\title{
Confinement Criterion for QCD with Dynamical Quarks
}

\author{
Klaus Fredenhagen \\ II. Institut für Theoretische Physik, Universität Hamburg, D-2000 Hamburg, West Germany \\ and \\ Mihail Marcu \\ Fakultät für Physik, Universität Freiburg, D-7800 Freiburg im Breisgau, Germany \\ (Received 28 January 1985)
}

We propose a criterion that tests the existence of isolated quarks in QCD.

PACS numbers: $12.38 . \mathrm{Gc}$

A long-standing open problem in QCD is the problem of quark confinement. In the limit of infinitely heavy (static) quarks, several confinement criteria have been proposed, the best known being that of Wilson. ${ }^{1}$ All of these criteria fail, however, in the presence of light (dynamical) quarks since vacuum fluctuations of quark-antiquark pairs screen the confining forces.

In experiments, quark confinement manifests itself in the fragmentation of sufficiently widely separated quark-antiquark pairs into ordinary hadrons. On the other hand, if free quarks are to exist it should be possible to separate a quark-antiquark pair. The free quark is then obtained by sending the antiquark to infinity. One is therefore led to investigate theoretically the sequence of "dipole" states

$$
\Phi_{\mathbf{x y}}=\sum_{i, \alpha, \beta} \psi_{i, \alpha}(\mathbf{x}) \bar{\psi}_{i, \beta}(\mathbf{y}) U_{\alpha \beta}\left(\mathscr{C}_{\mathbf{x y}}\right) \Omega,
$$

where $\psi_{i, \alpha}(\mathbf{x})$ are the quark fields at the point $\mathbf{x}$ of space with color index $\alpha$ and Dirac and flavor index $i$, $U\left(\mathscr{C}_{\mathbf{x y}}\right)$ is the path-ordered integral of the gauge field over the path $\mathscr{C}_{\text {xy }}$,

$$
U\left(\mathscr{C}_{\mathbf{x y}}\right)=\mathrm{P} \exp \left\{i \int_{\mathscr{C}_{\mathbf{x y}}} A\right\}
$$

and $\Omega$ is the vacuum.

The energy of the state $\Phi_{\mathbf{x y}}$ diverges as $|\mathbf{x}-\mathbf{y}|$ tends to infinity. In previous work $^{2-4}$ we discussed a possible way out of this difficulty. Denote by $U^{(n)}\left(\mathscr{C}_{x y}\right)$

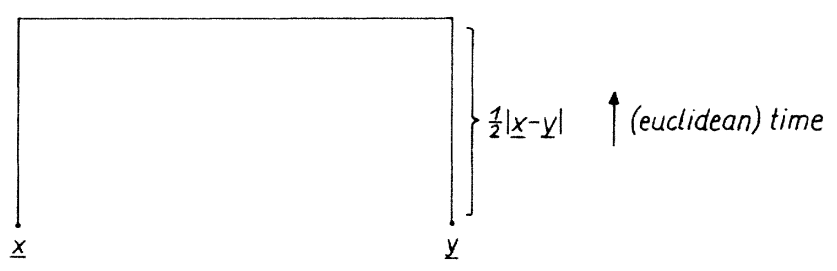

FIG. 1. The path $\mathscr{C}_{x y}$. the result of a translation of $U\left(\mathscr{C}_{\mathbf{x y}}\right)$ by $n$ steps into Euclidean time:

$$
U^{(n)}\left(\mathscr{C}_{\mathbf{x y}}\right)-T^{n} U\left(\mathscr{C}_{\mathbf{x y}}\right) T^{-n}
$$

Here $T$ is the transfer matrix. The energy of the sequence of states

$$
\Phi_{\mathbf{x y}}^{(n)}=\sum_{i, \alpha, \beta} \psi_{i, \boldsymbol{\alpha}}(\mathbf{x}) \bar{\psi}_{i, \boldsymbol{\beta}}(\mathbf{y}) U_{\alpha \beta}^{(n)}\left(\mathscr{C}_{\mathbf{x y}}\right) \Omega
$$

stays bounded as y goes to infinity if we take $n$ proportional to $|\mathbf{x}-\mathbf{y}|$. (This is a consequence of the perimeter law for the Wilson loop which is always true if the gauge fields are coupled to matter fields carrying the fundamental charge; see, e.g., Seiler ${ }^{5}$.) For the sake of simplicity we shall take $\mathbf{x}-\mathbf{y}$ along a lattice axis and choose $n=\frac{1}{2}|\mathbf{x}-\mathbf{y}|$. The Ansatz (4) mimics the attempt to separate a quark-antiquark pair with a given energy.

If quark fragmentation occurs as $\mathbf{y} \rightarrow \infty$, the transition probability of $\Phi_{x y}^{(n)}$ into hadronic states (including the vacuum) should go to 1 . In particular, since all hadronic states are local excitations of the vacuum, one expects

$$
\lim _{|\mathbf{x}-\mathbf{y}| \rightarrow \infty} \frac{\left|\left(\Omega, \Phi_{\mathbf{x y}}^{(n)}\right)\right|^{2}}{\left\|\Phi_{\mathbf{x y}}^{(n)}\right\|^{2}}=\mathrm{const} \neq 0 .
$$

If, on the other hand, the limit (5) is zero, this is an indication that the sequence of dipole states becomes orthogonal to all hadronic states and therefore approximates an isolated quark.

In order to compute the ratio in Eq. (5) we express the matrix elements in terms of Euclidean expectation values of gauge-invariant strings and loops. Denote by

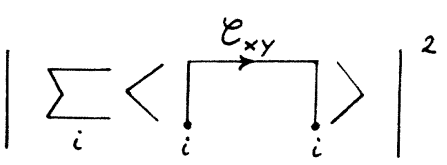

FIG. 2. The numerator of Eq. (5) in graphical terms. 


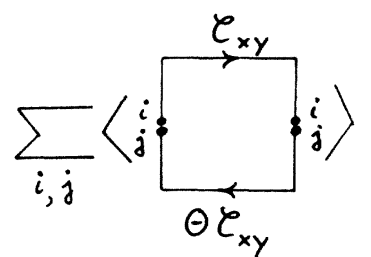

FIG. 3. The denominator of Eq. (5) in graphical terms.

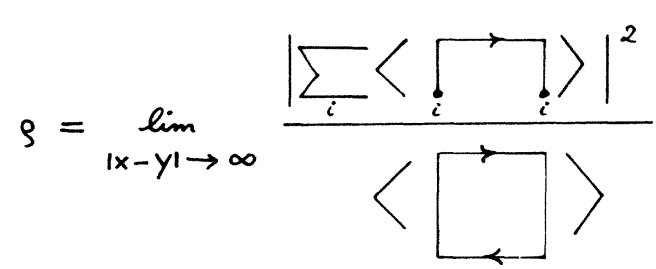

FIG. 4. The order parameter in graphical terms.
$\mathscr{C}_{x y}$ a rectangular path in Euclidean space-time with end points $\mathbf{x}$ and $\mathbf{y}$ in the time-zero hyperplane (Fig. 1). The numerator of (5) is given in graphical terms by Fig. 2 and the denominator is given by Fig. 3. In Fig. $3 \theta$ denotes "reflection through the time-zero hyperplane."

In the continuum theory, the denominator of Eq. (5) has to be regularized because of the singularity of products of quark fields at coinciding points. A simple way of doing this is to replace it by the expectation value of the Euclidean Wilson loop $\mathscr{C}_{x y} \cdot \theta \mathscr{C}_{x y}$. Thus the parameter to be tested can be represented in graphical terms by Fig. $4 .^{2}$ A regularization of the corners of the path $\mathscr{C}_{x y}$ is probably not necessary since the associated divergences in the numerator and denominator should cancel.

We would like to point out that our parameter $\rho$ tests in some sense the origin of the perimeter behavior of the Wilson loop in the presence of dynamical quarks. If this behavior is caused by fluctuations of the matter fields, then both the numerator and the denominator in Fig. 4 should decrease exponentially at the same rate. As a result $\rho \neq 0$, which means confinement. If, however, the perimeter behavior is partially due to fluctuations of the gauge field, the denominator is expected to decay more slowly. Then $\rho$ vanishes, which indicates deconfinement.

The picture described above has emerged from a general analysis of the particle structure in gauge theories, ${ }^{6}$ and has been confirmed both analytically ${ }^{3}$ and numerically ${ }^{4}$ on the example of the $Z_{2}$ Higgs theory. In the $Z_{2}$ model there exists both a confining and a deconfining phase, and $\rho$ shows the expected behavior.

As an aside we mention that our parameter looks similar to a parameter proposed by Bricmont and Fröhlich. ${ }^{7}$ However, the information contained in the latter work is different: The Bricmont-Fröhlich parameter is not sensitive to the presence of free quarks; it rather tests the existence of bound states of a dynamical and a static quark. ${ }^{3}$ Since the existence of bound states does not imply the absence of isolated quarks in the scattering states, the Bricmont-Fröhlich criterion is not directly relevant to the confinement problem.

For the application of our confinement criterion it is important to control the behavior of the parameter $\rho$ under renormalization-group transformations. Let $\rho=\rho(g)$ denote the dependence of $\rho$ on the coupling constant $g$ in massless QCD on a lattice. By imposition of a renormalization condition the lattice spacing $a$ is fixed as a function of $g$. The parameter $\rho$ in Fig. 4 should behave like a gauge-invariant two-point function $W_{2}(r, g)$ of the fields $\psi$ and $\bar{\psi}$ ( $r$ is the distance between $\mathbf{x}$ and $\mathbf{y}$ on the lattice) at a physical distance $r a$. The asymptotic behavior of $W_{2}(r, g)$ for small distances $r a$ can be determined from perturbation theory. The continuum value $\rho_{t}$ of our order parameter is obtained by taking the limit

$$
\rho_{c}=\left.\lim _{g \rightarrow 0} \frac{\rho(g)}{W_{2}(r, g)}\right|_{r a=\text { const }} .
$$

The parameter $\rho_{c}$ could be determined in a Monte Carlo simulation by computation of $\rho(g)$ and use of perturbative results for the renormalization-group equation and for $W_{2}(r, g)$. At a later stage the accuracy of the simulation could be improved to determine the scaling relations and the short-distance behavior of $W_{2}(r, g)$ directly from the simulation.

The parameter $\rho_{c}$ should be directly related to measured quantities in the quark fragmentation process. It would be very interesting to isolate such quantities, but this requires a more detailed study of the fragmentation process on the lattice.

We are indebted to Chris Korthals-Altes and Detlev Buchholz for helpful suggestions, and we would like to thank Peter Hasenfratz and Hartmann Römer for enlightening discussions. One of us (M.M.) would like to thank the Deutches Elektronen-Synchrotron theory group for its kind hospitality. One of us (K.F.) is the recipient of a Heisenberg fellowship.

${ }^{1}$ K. Wilson, Phys. Rev. D 10, 2445 (1974).

${ }^{2} \mathrm{~K}$. Fredenhagen, talk presented at the Colloquium in honor of Professor Haag on the occasion of his 60th birthday, Hamburg, November 1982, University of Freiburg Report No. THEP 82/9 (unpublished).

${ }^{3}$ K. Fredenhagen and M. Marcu, Commun. Math. Phys. 92, 81 (1983).

${ }^{4} \mathrm{~K}$. Fredenhagen and M. Marcu, to be published.

${ }^{5} \mathrm{E}$. Seiler, Gauge Theories as a Problem of Constructive Quantum Field Theory and Statistical Mechanics, Springer Lecture Notes in Physics Vol. 159 (Springer, Berlin, 1982).

${ }^{6} \mathrm{D}$. Buchholz and K. Fredenhagen, Commun. Math. Phys. 84, 1 (1982).

7J. Bricmont and J. Fröhlich, Phys. Lett. 122B, 73 (1983). 